\title{
Application of microbiological assay to determine the potency of intravenous antibiotics
}

\author{
Saimun Nahar, Most. Salma Khatun and Md. Shahidul Kabir* \\ Department of Microbiology, Stamford University Bangladesh, 51, Siddeswari Road, Dhaka-1217, Bangladesh
}

Received 20 Nov 2020/Accepted 04 Dec 2020

\begin{abstract}
Demonstration of equivalent amounts of active pharmaceutical ingredient is a basic requirement for intravenous generic drugs prior to administration. Physicochemical methods are often used to determine concentration of antibiotics in biological fluids. However, it does not permit direct quantification of potency of a desired antibiotic. This study demonstrates the application of a microbiological assay to determine the potency and concentration of commercially available pharmaceutical-grade antibiotics used for injections. Concentration-dependent variation of inhibitory effect of four commercial brands of cefuroxime and two of ciprofloxacin were observed against two reference bacteria (Escherichia coli DH5a and Escherichia coli ATCC 8739) on Mueller Hinton agar. Regression analysis was used to assess the in vitro equivalence of generic products sold by different retail companies in Dhaka city. A linear relationship was found between the concentration and response of the bacteria in regression analysis where anti-log of $X$-intercept and slope showed the concentration and potency, respectively. The study showed excellent results of linearity $\left(r^{2} \geq 0.89\right)$, precision (inter assay variation $\leq \mathbf{1 0 \%}$ for cefuroxime and $\leq \mathbf{2 0} \%$ for ciprofloxacin), accuracy and specificity tests for both types of antibiotics. Pharmaceutical equivalence demonstrated by four cefuroxime and two ciprofloxacin samples showed no significantly distinguishable slopes $(P>0.78$ and $P>0.44)$ and intercepts $(P>0.25$ and $P>0.07)$, respectively. Estimated potency for cefuroxime was 91.1-100.0\% and for ciprofloxacin was 68.1-100.0\%. Microbiological assay was found to be convenient, rapid, cost-effective, precise and accurate in demonstrating pharmaceutical equivalence of antibiotics in different dosage forms. This technique can be used as an alternative method for testing generic antibiotics prior to their use in animal and human.
\end{abstract}

Keywords: Antibiotics, Microbiological assay, Potency.

\section{INTRODUCTION}

Antibiotics are chemicals that are used in low amount to kill or inhibit the growth of microorganisms in human and animals. Irrespective of global increase of antibiotic resistance, widespread use of these drugs still continues to be a major threat to the safety of human and animal lives. Antibiotics are either inappropriately prescribed by physicians or purchased by individual over the counter for treatment of infectious diseases. Antibiotics are widely used as anti-infectives and over half of the prescriptions include at least one generic product $(1,2)$. Antibiotics account for over $50 \%$ of sales which comprise a market of US\$66.5 billion worldwide (3). There is a phenomenal growth in the production of such generic compounds by a range of pharmaceutical companies worldwide and in most cases they do not require to demonstrate therapeutic efficacy (4-5). In most cases leading drug producers only maintain the pharmaceutical equivalence of same active chemicals of same dosage forms for manufacture under environment prescribed in the standard specifications (6). It is therefore necessary to quantify active pharmaceutical ingredients (API) in different dosage forms during and after production. Several chemical methods such as, High Performance Liquid
Chromatography (HPLC), UV spectrophotometry, immunological assays (fluorescence polarization immunoassay) and radioimmunoassay are used to determine the potency of API (7). For some antibiotics chromatographic methods are preferred to determine the exact concentration of active ingredients. However, antibiotics are often adulterated and faked by several manufacturers during production which is a threat for effective treatment and will give rise to problem of super-infections caused by resistant bacteria $(8,9,10)$. Microbiological methods can precisely determine both potency and bioactivity of an antibiotic which is a limitation for chemical assays. Microbiological assays are simple, do not require specialized instruments and toxic chemicals, hence convenient for determining potency of antibiotics in a laboratory $(11,12)$.

Microbiological assay was first observed by Fleming which was lysozomal activity in agar plate and this assay was shortly followed by Heatley in an agar diffusion assay for penicillin (13). A reduction in the antimicrobial activity can be easily determined by microbiological assay under standard environmental condition which may demonstrate subtle change by chemical methods $(14,15)$. Microbiological assay can be carried out under standard condition to observe the inhibition of growth of fully characterized microbial 
strains against different concentrations of antibiotics (16). Determination of potency of antibiotics is an essential criterion for the quality control and quality assurance of antibiotic preparation $(17,18)$. Different microbiological assays were prescribed by scientists where potency cannot be adequately determined by chemical assays viz. Kirby-Bauer disk diffusion method (19), E-test (Epsilometer test) (20), agar diffusion method (Cylindrical-plate or Cup-plate) (21, 22 ) etc. In this study, two known bacterial strains were used in agar plate assay to determine the potency and concentration of cefuroxime and ciprofloxacin.

\section{MATERIALS AND METHODS}

Antibiotics. Intravenous antibiotics for human use were bought from reputable local drug stores for this study. All products were licensed under the drug regulatory agency of Bangladesh. We used four samples of Cefuroxime $(\mathrm{s} 1, \mathrm{~s} 2, \mathrm{~s} 3, \mathrm{~s} 4)$ and two samples of Ciprofloxacin $(\mathrm{s} 1, \mathrm{~s} 2)$ in this study. All products were reconstituted with calibrated micropipettes following the manufacturers' instruction for assay purpose. Pure-grade reference powders of both antibiotics were used as controls and procured from Sigma Aldrich, St Louis, MO. The former were weighted in an analytical balance and the latter was diluted to a final concentration of cefuroxime $75 \mu \mathrm{g} / \mu \mathrm{l}$ and ciprofloxacin $2 \mu \mathrm{g} / \mu \mathrm{l}$ in distilled water. Pure-grade reference powder for both cefuroxime and ciprofloxacin were used as control. Media, strains and inocula. Testing strains were Escherichia coli ATCC 8739 and Escherichia coli DH5 $\alpha$ for cefuroxime (s1, s2, s3 and s4) and ciprofloxacin (s1 and s2). To grow E. coli ATCC 8739 and E. coli DH5 $\alpha$, we followed standard protocols (23). Briefly, each bacterial stock previously stored and frozen at $-20^{\circ} \mathrm{C}$ was resuscitated on solid medium (in two successive Trypticase Soy Agar) and five colonies were selected and passed to $10 \mathrm{ml}$ of Nutrient Broth. After overnight incubation at $37^{\circ} \mathrm{C}$, we made a second transfer from the last tube with visible growth into $10 \mathrm{ml}$ of fresh liquid medium and again incubated for 6 hours at $37^{\circ} \mathrm{C}$.

Glass-plates assay. Large glass plates/petri plates were used to run al assays in duplicates of different generic products on Mueller Hinton agar media (24). Boring devices were used to make well of 6 millimeter onto plates for different concentrations of generic products and known antibiotics powders. The device was routinely cleaned (iodine soap and water), disinfected with $70 \%$ ethanol and flamed to sterilize. Suspensions of $E$. col ATCC 8739 and $E$. coli DH5 $\alpha$ having 0.5 MacFarland standard were used to inoculate the surface of the plate and dried for 15-20 min. Different concentrations of antibiotics were added to each well in duplicate and incubated at $37^{\circ} \mathrm{C}$ for overnight. Diameters of zone of inhibition in millimeters were measured and plotted against different concentrations.
Statistical analyses. The linearity, precision, accuracy and specificity were determined to validate method for testing pharmaceutical equivalence. Logarithm value of concentration (x-axis, $\log 10 \mathrm{mg} / \mathrm{l}$ ) of each sample was plotted against their respective zones of inhibition (y-axis, mean diameter in $\mathrm{mm})$. Seven point standard curves were prepared using different concentrations of known antibiotic powders of ciprofloxacin and cefuroxime. Similar curves were also prepared for with the generic drugs from different companies. The range of different concentrations those were used viz., 18, $16,14,12,10,8,6 \mu \mathrm{g} / \mu \mathrm{l}$ for Ciprofloxacin and 32, 24, 16, 8, 4, 1, $0.25 \mu \mathrm{g} / \mu \mathrm{l}$ for Cefuroxime respectively. Data were expressed in a linear model $(\mathrm{y}=\mathrm{mx}$ $+b$, where $b$ is the $y$-intercept intercept and $m$ is the slope) best fitting the data in regression analysis (Microsoft Office Excel 2010) with 95\% confidence intervals (CI) (25). Linearity was expressed as coefficient of variation $\left(\mathrm{r}^{2}\right)$ and standard error was estimated. Symmetrical parallel-line assay was used to test pharmaceutical equivalence comparing slope and intercept of each generic product with those of the known powder by overall test for coincidence of the regression lines, a statistical technique for Curve Fitting Analysis (CFA) (26). The slope of the linear regression was defined as potency and the anti-log of $\mathrm{x}$-intercept was calculated as concentration when $\mathrm{y}=0$. When $\mathrm{y}$ is at the mid-point of the linear regression, relative concentration was estimated at $95 \%$ confidence interval of the predicted line. Intercepts and slopes of linear regressions produced by the reference standard and each extra group were compared by Curve Fitting Analysis (CFA) (27).

Assuming that generic and raw powders are the same product, a pharmaceutically equivalent generic must display a parallel and overlaid curve with respect to the known antibiotic powder $(\mathrm{P}>0.05)$. Parallel curves of same concentrations of antibiotics will superimpose one another and will demonstrate same intercept. Lack of parallelism indicates different generic products. However parallel curve with different intercepts will indicate identical APIs with either higher or lower concentrations. As per the international pharmacopoeias, the relative potency of each generic to known antibiotic powder was calculated as the $\mathrm{x}$-distance between the two lines (28). The repeatability of the assay was determined using a minimum of three concentrations of each antibiotic by triplicates during the same day or under similar experimental conditions but with different biological matrices and plates (intra-assay precision), and comparing the results of assays on different days (inter-day precision). These were expressed as means with standard deviations and coefficients of variation (CV) (29).

\section{RESULTS}

\section{Curve fitting analysis of the linear regression model to data.}

Figure 1 shows the linear regression analysis of logarithm value of concentration ( $\mathrm{x}$-axis, $\log 10 \mathrm{mg} / \mathrm{l}$ ) of each sample against their respective zones of inhibition (y-axis, mean diameter in $\mathrm{mm}$ ). The best straight line predicted from data obtained by microbiological assay for the generic antibiotics

Table 1. Potency estimate derived from linear regression analysis with their statistical comparison of generics versus known antibiotics.

\begin{tabular}{|c|c|c|c|c|c|c|c|c|}
\hline \multicolumn{3}{|c|}{ Products } & \multirow{2}{*}{$\begin{array}{c}\mathbf{r}^{2} \\
\text { (linearity) }\end{array}$} & \multirow{2}{*}{$\begin{array}{l}\text { Intercept } \\
\quad \text { (C) }\end{array}$} & \multirow{2}{*}{$\begin{array}{c}\text { P value } \\
\text { (concentration) }\end{array}$} & \multirow{2}{*}{$\begin{array}{c}\text { Slope } \\
(95 \% \mathrm{CI})\end{array}$} & \multirow{2}{*}{$\begin{array}{c}\text { P- } \\
\text { value for } \\
\text { potency }\end{array}$} & \multirow{2}{*}{$\begin{array}{c}\text { Potency } \\
\text { Estimate (\%) }\end{array}$} \\
\hline \multicolumn{2}{|c|}{ Antibiotics } & Isolates & & & & & & \\
\hline \multirow{7}{*}{ 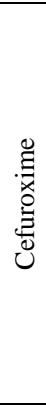 } & $\mathrm{S} 1$ & \multirow{3}{*}{ 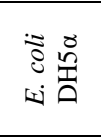 } & 0.92 & 35.50 & 0.784 & 8.508 & \multirow{7}{*}{$\mathrm{p}<0.05$} & $98.20 \%$ \\
\hline & Fig: e & & & & \multirow{6}{*}{$\mathrm{p}<0.05$} & & & \\
\hline & $\begin{array}{c}\text { S2 } \\
\text { Fig: } \mathrm{f}\end{array}$ & & 0.92 & 35.24 & & 7.930 & & $92.42 \%$ \\
\hline & $\begin{array}{c}\text { S1 } \\
\text { Fig: a }\end{array}$ & \multirow{4}{*}{ 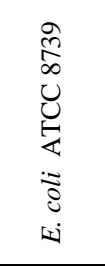 } & 0.89 & 13.98 & & 9.593 & & $99.10 \%$ \\
\hline & $\begin{array}{c}\text { S2 } \\
\text { Fig: b }\end{array}$ & & 0.89 & 14.35 & & 8.748 & & $91.1 \%$ \\
\hline & $\begin{array}{c}\text { S3 } \\
\text { Fig: c }\end{array}$ & & 0.91 & 13.43 & & 8.49 & & $91.1 \%$ \\
\hline & $\begin{array}{c}\text { S4 } \\
\text { Fig: d }\end{array}$ & & 0.895 & 13.12 & & 9.372 & & $97.7 \%$ \\
\hline \multirow{4}{*}{ 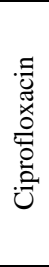 } & $\begin{array}{c}\text { S1 } \\
\text { Fig: g }\end{array}$ & \multirow{2}{*}{ 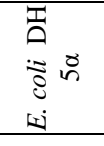 } & 0.95 & 16.53 & 0.438 & 18.33 & \multirow{4}{*}{$\mathrm{p}<0.05$} & $98.80 \%$ \\
\hline & $\begin{array}{c}\text { S2 } \\
\text { Fig: } h\end{array}$ & & 0.90 & 23.90 & $\mathrm{p}<0.05$ & 12.48 & & $68.1 \%$ \\
\hline & $\begin{array}{c}\text { S1 } \\
\text { Fig: i }\end{array}$ & \multirow{2}{*}{ 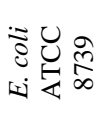 } & 0.97 & 4.25 & & 14.72 & & $99.02 \%$ \\
\hline & $\begin{array}{c}\text { S2 } \\
\text { Fig: } j\end{array}$ & & 0.97 & 7.78 & & 11.93 & & $81.0 \%$ \\
\hline
\end{tabular}


cefuroxime and ciprofloxacin are shown in Figure 1. All cases exhibited a linear relationship between the logarithm of the concentration $(\log 10 \mathrm{mg} / \mathrm{l})$ and the diameter $(\mathrm{mm})$ of the zones of inhibition with high coefficients of determination $\left(\mathrm{r}^{2} \geq 0.89\right)$, low standard errors of the estimate (Syx $\leq 0.45)$ and statistically significant intercept and slope ( $\mathrm{P}<0.05$ by ANOVA). Determination of pharmaceutical equivalence The log concentration-response relationships of all generic products and reference powders were parallel and overlaid curve without significant difference by CFA ( $\mathrm{P} \geq 0.89$, Table 2), demonstrating that all

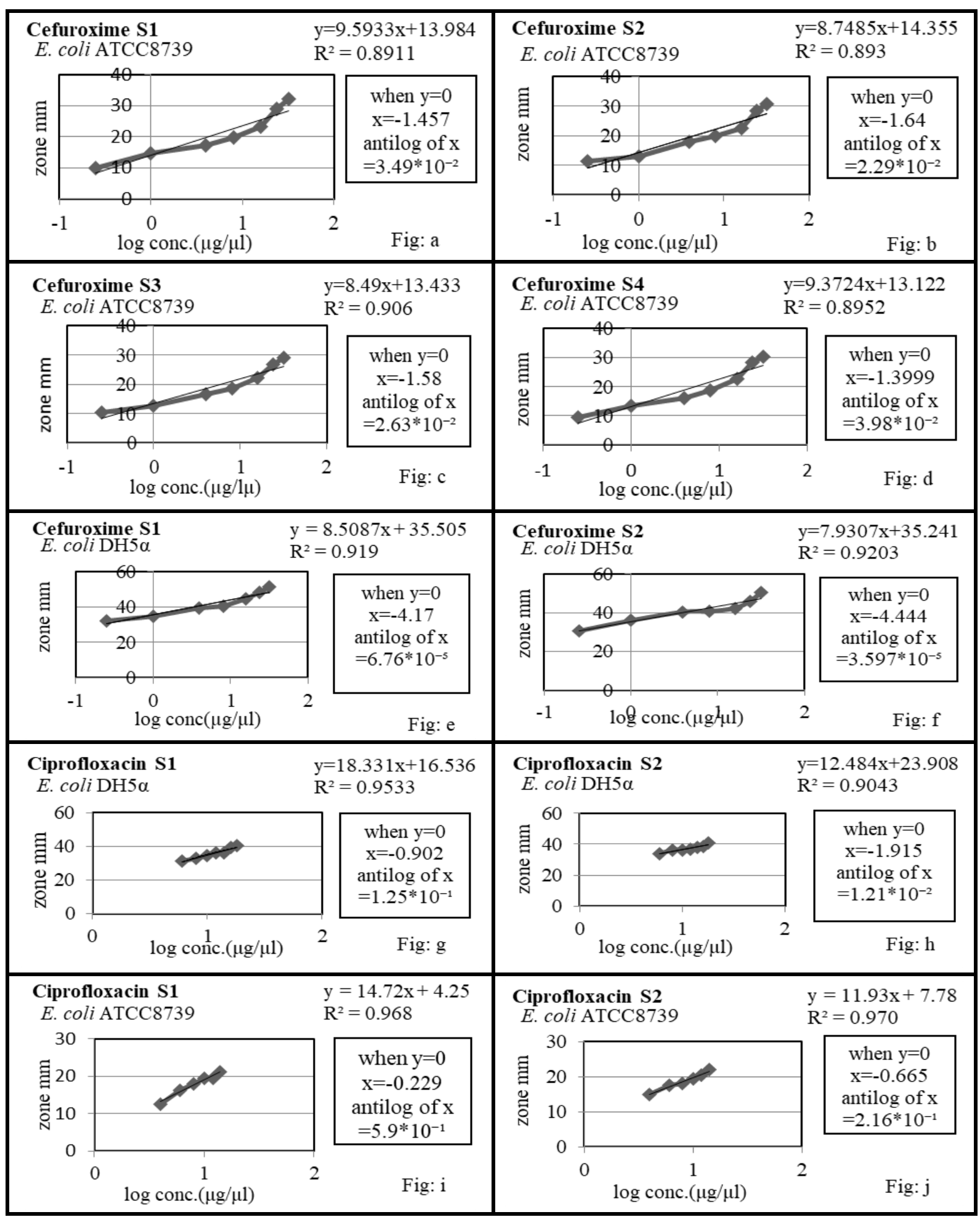

Figure 1. Multiple linear curves of cefuroxime (s1, s2, s3 and s4) and ciprofloxacin (s1 and s2) for E. coli ATCC 8739 are shown in Figure 1 (a,b,c and d) and (i and j), respectively. Other linear curves of cefuroxime (s1 and s2) and ciprofloxacin (s1 and s2) for E. coli DH5 $\alpha$ are shown in Figure 1 (e and $\mathrm{f}$ ) and ( $\mathrm{g}$ and $\mathrm{h}$ ), respectively. 
products had the same biologic activity (potency) and concentration (intercept). The potency estimate of generic products ranged from 91.10 to $99.10 \%$ for cefuroxime and 68.10 to $99.02 \%$ for ciprofloxacin (Table 1).

\section{DISCUSSION}

Antibiotics are chemotherapeutic agents which are used in low concentration against pathogenic bacteria to control spread of infections caused by them (30, 31). Antibiotics are chemicals that are either produced by microorganisms or synthesized chemically are used against pathogenic bacteria. As they are applied in low concentrations to human and animals, a mild variation in the concentrations can affect their efficacy $(32,33)$. Therefore it is necessary to quantify active ingredients in antibiotic samples during and after production. Both chemical and biological methods can be used for such quantification (32), but microbiological method assay can be used to determine both potency and bioactivity of antibiotics. Although subtle change in antimicrobial activity can be demonstrated by chemical methods loss of activity of antibiotics can only be determined by standard methods like microbiological assay (34).

Table 1 shows relevant information of ciprofloxacin and cefuroxime tested in this study. All generic products were manufactured outside Bangladesh and legally imported for clinical use. Figure 1 a-j shows the $\log$ concentration-response relationship and the best straight line predicted from data obtained by microbiological assay for the ciprofloxacin and cefuroxime. All cases exhibited a linear relationship between the logarithm of the concentration $\left(\log _{10} \mu \mathrm{g} / \mu \mathrm{l}\right)$ and the diameter $(\mathrm{mm})$ of the zones of inhibition with high coefficients of determination $\left(\mathrm{R}^{2}\right.$ $\geq 0.891$ ), low standard errors of the estimate (Syx $\leq$ $0.358)$ and statistically significant intercept and slope ( $\mathrm{P}>0.78$ and $\mathrm{P}>0.44 ; \mathrm{P}>0.25$ and $\mathrm{P}>0.07$, respectively). Potency estimates were 92.42 to $100.0 \%, 91.1$ to $100.2 \%$ and 68.1 to $99.9 \%, 81.0 \%$ to $100 \%$ for generic products of cefuroxime and ciprofloxacin, respectively.

Kirby-Bauer disk diffusion and broth dilution methods are used to determine the sensitivity and resistance of bacteria isolated from either clinical or environmental sources. In Kirby-Bauer method a lawn of test microorganisms are prepared on MuellerHinton agar and filter paper discs having different concentrations of antibiotics are paced at certain distance on the plates. After overnight incubation the zone of inhibition around the antibiotic discs indicates the ability of the antibiotics to kill microorganisms (19). In this method only the effect of antibiotics are measured but the minimum inhibitory concentration is not measured. However, in broth dilution method, different concentrations of antibiotics are added to tubes containing Mueller-Hinton broth. Tube containing the lowest concentration of antibiotic that inhibit the growth of microorganisms is considered as minimum inhibitory concentration (MIC). In agar dilution method an antimicrobial agent is incorporated into solid agar medium in a petri dish. A standard amount of microorganisms is inoculated onto the surface of the medium and allowed to grow overnight. Like the broth dilution method the minimum concentration at which the growth of the test microorganisms is inhibited is called MIC of the antibiotics used (19).

In E-test (Epsilometer test), antimicrobial susceptibility of the test organism is determined against a specific antibiotic. Commercially prepared filter paper strip is impregnated with a gradient of concentration of antibiotic. Such E-test strip is applied onto an inoculated agar plate. The intersection of inhibitory zone edge and the E-test strip indicates the MIC value. This test is considered as more accurate and precise than previously mentioned tests (35).

Agar diffusion method is a well known approach where cylindrical-plate or cup-plates are used to estimate the potency and bioactivity of antibiotics. Antibiotics diffuse from the cup or cylinder to the outer environment in the plate. Based on the gradient of concentrations of antibiotics zones of inhibition are formed around these cups or cylinders. The zones of inhibition at different dosages are compared to the standard to determine similar biological effect in the sample (34).

A number of factors can influence the variability and error in microbiological assay. For example, in disc diffusion assay unequal exposure of the plates placed in a stack in incubator, variation of time between pouring seeded agar in the plates and time of applying solution of antibiotics to the plates (36). Variation in temperature within the stack can be minimized by enclosing the stack in close-fitting steel cylinders. Thickness of agar, concentration of inoculums, incubation temperature, exposure time, $\mathrm{pH}$, chemical composition of media and $\mathrm{pH}$ of buffer solution can be among other factors to be considered $(30,32)$. The zones of inhibition will be reduced with the increase in the thickness of the agar media $(30,32,37)$. These experimental variations for incubation temperature, time can be minimized by arranging multiple disc or cylinders in the same petri dish.

\section{CONCLUSION}

Antibiotics are routinely used for treatment of infectious diseases caused by bacteria. However, most of the cases these are wrongly prescribed or misused and now become the most serious problems all over the world. Scientists are trying relentlessly in different ways and means to overcome this problem. To order to overcome this problem of antibiotic resistance it is necessary to correctly determine the potency and bioactivity of antibiotics. A microbiological assay is always more convenient over those like HPLC which needs sophisticated instruments and skilled manpower to do the assay. Although HPLC is a rapid, accurate 
and precise in terms of detecting potency of antibiotic but it cannot determine bioactivity of antibiotic. Microbiological assays are rapid, accurate and precise for determination of both potency and bioactivity. Although there are some biological errors in microbiological assay these assay these can be reduced through appropriate validation.

\section{REFERENCES}

1. Gerbino PP and Joseph AS. 1993. Multisource drugs: implication and concerns in the geriatric population. Hosp. Pharm. 28:96-2

2. Marketing Authorization of Pharmaceutical Products with Specia Reference to Multisource (Generics) Products: A Manual for Drug Regulatory Authorities [http://www.who.int/ prequal/ info_general/documents/WHO_DMP RGS 98 5 R.pdf]

3. The Global Antibacterials Market: R \& D Pipelines, Market Analysi and Competitive Landscape. Arrowhead Publishers. 2007:1-126. [http:www.arrowheadpublishers.com/ reports/infectious-disease/ theglobal-antibacterials-mark/\#].

4. Kirking DM and Ascione FJ. 2001. Perspectives on generic pharmaceuticals: some conclusions. J. Am. Pharm. Assoc (Wash). $41: 826-828$.

5. Dettelbach HR. 1986. A time to speak out on bioequivalence and therapeutic equivalence. J. Clin. Pharmacol. 26:307-308.

6. Welage LS, Kirking DM, Ascione FJ and Gaither CA. 2001 Understanding the scientific issues embedded in the generic drug approval process. J. Am. Pharm. Assoc (Wash). 41: 856-867.

7. Pfaller MA, Krogstad DJ, Granich GG and Murray PR. 1984 Laboratory evaluation of five assay methods for vancomycin: bioassay, high-pressure liquid chromatography, fluorescence polarization immunoassay, radioimmunoassay and fluorescence immunoassay. J. Clin. Microbiol. 20:311-316.

8. Ejikeme UC and Ademola OJ. 2010. Microbiological assay of the active component of ampicillin in ampicillin and ampicillin/cloxacillin suspensions using Bacillus megatharium NCTC 10342A (76) as indicator organism. Afr. J. Microbiol. Res.4:51-54.

9. Dafale NA, Semwal UP, Agarwal PK, Sharma P and Singh N. 2015. Development and validation of microbial bioassay for quantification of levofloxacin in pharmaceutical preparations. J. Pharm. Anal. 5:1826.

10. Prescott LM, Harley JP and Klein DA. 2008. Microbiology, Seventh ed., Mcgraw-Hill, New York. pp.835-858.

11. Pinto TJA, Lourenco FR and Kaneko TM. 2007. Microbiologica assay of gentamycin employing an alternative experimental design, AOAC Annual Meeting and Exposition, Anais. Anahein-California pp. 157 .

12. Saviano AM, Francisco FL and Lourenco FR. 2014. Rational development and validation of a new microbiological assay for linezolid and its measurement uncertainty. Talanta. 127:225-229.

13. Heatley NG. 1944. A method for the assay of penicillin. Biochem J. 38:61-65.

14. Abdelaziz AA, Elbanna TE and Gamaleldeen NM. 2012. Validated microbiological and HPLC methods for the determination of moxifloxacin in pharmaceutical preparations and human plasma. Braz. J. Microbiol. 43:1291-1301.
15. United States Pharmacopoeia, United States Pharmacopoeial Convention, Rockville, MD, U.S.A. 2009. pp.86-93.

16. United States Pharmacopoeia, United States Pharmacopoeial Convention, Rockville, MD, U.S.A. 2009. pp. 86-93

17. Yamamoto $\mathrm{CH}$ and Pinto TJA. 1996. Rapid determination of neomycin by a microbiological agar diffusion assay using triphenyl tetrazolium chloride. J. Assoc. Anal. Chem. 79:434-440.

18. Lourenco FR and Pinto TJA. 2009. Comparison of three experimental designs employed in gentamycin microbiological assay through agar diffusion. Braz. J. Pharm. Sci. 45:559-566.

19. Prescott LM, Harley JP and Klein DA. 2008. Microbiology, Seventh ed., Mcgraw-Hill, New York., pp.835-858.

20. Black JG. 2005. Microbiology: Principles and Explorations, Sixth ed., John Wiley \& Sons Inc., USA, pp. 352-384.

21. Indian Pharmacopoeia, Indian Pharmacopoeia Commission, Ghaziabad, India, 2014, pp.50-59.

22. British Pharmacopoeia, The Stationary Office, London, 2015, pp. V396-V402.

23. Isenberg HD: Clinical Microbiology Procedures Handbook. Washington DC, USA, ASM Press; 2004.

24. Bennett JV, Brodie JL, Benner EJ and Kirby WM. 1966. Simplified, accurate method for antibiotic assay of clinical specimens. Appl. Microbiol. 14:170-177.

25. Mark H. 2003. Application of an improved procedure for testing the linearity of analytical methods to pharmaceutical analysis. J. Pharm. Biomed. Anal. 33:7-20.

26. Glantz SA. 2006. Primer of Biostatistics, New York, McGraw-Hill Companies.

27. Food and Drug Administration. 2001. Guidance for Industry: Bioanalytical Method Validation. Rockville.

28. United States Pharmacopeia Convention. 2002. United States Pharmacopeia. Rockville, MD.: United States Pharmacopeia Convention. 1083-1084.

29. Petersen HH, Nielsen JP, Jensen AL and Heegaard PM. 2001. Evaluation of an enzyme-linked immunosorbent assay for determination of porcine haptoglobin. J. Vet. Med. A Physiol. Pathol. Clin. Med. 48:513-523.

30. Dafale NA, Semwal UP, Agarwal PK, Sharma P and Singh GN. 2012. Quantification of ceftriaxone sodium in pharmaceutical preparations by new validated microbiological bioassay. Anal. Methods. 4:24902498.

31. Denyer SP, Hodges NA and Gorman SP. 2004. Hugo \& Russell's Pharmaceutical Microbiology, Seventh ed., Blackwell Publishing Company, UK,

32. Dafale NA, Semwal UP, Agarwal PK, Sharma P and Singh GN. 2015. Development and validation of microbial bioassay for quantification of levofloxacin in pharmaceutical preparations, J. Pharm. Anal. 5:1826.

33. Hewitt W. 1977. Microbiological Assay: An Introduction to Qualitative Principles and Evaluation, First ed., Academic Press, New York, pp. 1-50.

34. United States Pharmacopoeia. 2009. United States Pharmacopoeial Convention, Rockville, MD, U.S.A, pp.86-93.

35. Black JG. Microbiology: Principles and Explorations, Sixth ed., John Wiley \& Sons Inc., USA, 2005, pp.352-384.

36. Davis WW and Stout TR. 1971. Disc plate method of microbiological antibiotic assay factors influencing variability and error-1. Appl. Microbiol. 22:659-665.

37. Dafale NA, Agarwal PK, Semwal UP and Singh GN. 2013. Development and validation of microbial bioassay for the quantification of potency of the antibiotic cefuroxime axetil. Anal. Methods. 5:690-698. 